\title{
Robot-Assistant for MRI-Guided Liver Ablation: \\ a pilot study
}

Enrico Franco a) and Mike Ristic

Mechanical Engineering department, Imperial College London, London SW7 AZ, United

Kingdom

Marc Rea and Wladyslaw M. W. Gedroyc

Department of radiology, Imperial College Healthcare NHS Trust, London W2 1NY, United Kingdom

a) Author to whom correspondence should be addressed. Electronic mail: ef1311@imperial.ac.uk

Purpose: Percutaneous ablation under MRI-guidance allows treating otherwise inoperable liver tumors locally using a catheter probe. However, manually placing the probe is an error-prone and time consuming task that requires a considerable amount of training. The aim of this paper was to present a pneumatically actuated robotic instrument that can assist clinicians in MRI-guided percutaneous intervention of the liver and to assess its functionality in a clinical setting. The robot positions a needle-guide inside the MRI scanner bore and assists manual needle insertions outside the bore.

Methods: The robot supports double oblique insertions that are particularly challenging for less experienced clinicians. Additionally, the system employs only standard imaging sequences and can therefore be used on different MRI scanners without requiring prior integration. The repeatability and the accuracy of the robot were evaluated with an optical tracking system. The functionality of the robot was assessed in an initial pilot study on two patients that underwent MRI-guided laser ablation of the liver.

Results: The robot positioned the needle-guide in a repeatable manner with a mean error of $0.35 \mathrm{~mm}$ and a standard deviation of $0.32 \mathrm{~mm}$. The mean position error corresponding to the needle tip, measured for an equivalent needle length of $195 \mathrm{~mm}$ over 25 fixed points, was $2.5 \mathrm{~mm}$ with a standard deviation of $1.2 \mathrm{~mm}$. The pilot study confirmed that the robot does not interfere with the equipment used for MRI-guided laser ablation and does not visibly affect the MR images. The robot setup integrated 
seamlessly within the established clinical workflow. The robot-assisted procedure was successfully completed on two patients, one of which required a complex double oblique insertion. For both patients, the insertion depth and the tumor size were within the range reported for previous MRI-guided percutaneous interventions. A third patient initially enrolled in the pilot study was considerably heavier than the others, preventing the use of the robot and requiring several freehand insertion attempts.

Conclusions: The robot repeatability and accuracy are appropriate for liver tumors normally treated with MRI-guided ablation. The results of the pilot study endorse the clinical use of the robot in its current form: the robot is fully functional and MRIcompatible in a clinical setting and is suitable for double-oblique needle insertions.

Key words: Medical robot, Magnetic Resonance Imaging, image-guided therapy, liver ablation

\section{INTRODUCTION}

Percutaneous laser ablation employs a water-cooled applicator to deliver thermal energy generated by a high-power laser source directly to the lesion, allowing to treat otherwise inoperable liver tumors. ${ }^{1}$ Typically, a coaxial needle is initially inserted in the lesion location under either ultrasound, CT, or MRI guidance and then replaced with the laser applicator. ${ }^{1,2}$ Compared to ultrasound or CT guidance, MRI offers higher softtissue contrast and allows thermal monitoring during the ablation. ${ }^{3}$ Recent research suggests that specially designed microcoils could further enhance the temperature accuracy during MRI-guided ablation therapies. ${ }^{4}$ While open MRI scanners could allow simultaneous imaging and needle insertion, they are less common in hospitals and have limited imaging capabilities compared to closed-bore scanners. ${ }^{2}$ Conversely, needle insertions in closed-bore scanners entail repeatedly moving the patient in and out of the bore because of the space constraints. ${ }^{5}$ Additionally, conventional freehand needle

55 insertions require considerable training and $2 \mathrm{D}$ to $3 \mathrm{D}$ extrapolation skills. Consequently, several insertion attempts may be necessary. ${ }^{6}$ This results in time consuming procedures that can last over 2 hours. $^{7}$ 
Specially designed robots can potentially benefit MRI-guided percutaneous interventions in terms of more accurate and faster targeting. However, the MRI environment poses severe restrictions on the choice of materials, actuators, and sensors, while the space constraints of closed-bore scanners demand compact designs. A first needle-guiding robot for microwave ablation of the liver in open MRI scanners that employs ultrasonic actuators was presented in ${ }^{8}$. Ultrasonic motors have been used since then in robots for MRI-guided brain intervention, ${ }^{9}$ gynecologic intervention, ${ }^{10}$ 65 prostate intervention, ${ }^{11,12}$ and for more general research purposes. ${ }^{13}$ However they have been shown to produce image degradation in some conditions and should therefore be employed with appropriate shielding and kept outside of the imaging volume. ${ }^{14}$ Manually actuated manipulators intended for closed-bore scanners were developed as a cost effective alternative. ${ }^{15,16}$ Although manual actuation does not degrade the MR images, it requires long transmissions with inherent problems of friction, backlash, limited bandwidth, and limited accuracy. Pneumatic actuation instead can qualify as MRI-safe ${ }^{17}$ and offers a comparatively affordable alternative to ultrasonic motors. ${ }^{18}$ Consequently, it has been successfully employed for MRI-guided biopsy and intervention on the prostate, ${ }^{19,20}$ the breast, ${ }^{21,} 22$ the spinal cord, ${ }^{23}$ and for functional-

75 MRI. $^{24}$ While accurate position control is the main challenge associated with pneumatic actuation, high accuracy was achieved with Time Delay Control algorithms (TDC). ${ }^{25}$

The space constraints of closed-bore scanners are particularly limiting for robotic devices operating on the patient's torso. In such case, a robot structure consisting of a gantry mounted on the scanner bed and supporting an actuated arm has generally been

80 preferred. ${ }^{16,23} \mathrm{~A}$ body-mounted robot for percutaneous intervention with a compact footprint has recently been proposed as a promising alternative. ${ }^{26}$ However, the robot has to be manually placed on the correct incision site, which is assumed known from preoperative images. Additionally, the access to the incision site and the concomitant presence of radio-frequency $(\mathrm{RF})$ receiver coils on the patient's abdomen remain potentially problematic with this architecture. Overall, while a variety of designs and actuation strategies have been developed for different MRI-guided procedures, to the best knowledge of the authors, only very few systems have been validated in a clinical setting. ${ }^{27}$ 
This work presents a robotic instrument designed to assist clinicians in MRI-guided

ablations of liver tumors and reports an initial pilot study on two patients. The robot employs pneumatic actuation and is powered from outside the scanner room in order to minimize the interference to the MRI environment. Differently from ${ }^{8}$, the robot was designed to operate in closed-bore MRI scanners. Differently from ${ }^{26}$, the robot can realign to different incision sites without requiring manual repositioning, offering more

flexibility in a clinical setting. Compared to our initial prototype ${ }^{25}$ this system has a larger workspace, a more elaborated control strategy, and a refined user interface. The robot design, the kinematics, the control strategy, and the clinical workflow are outlined in the following sections. The repeatability and the accuracy of the robot were assessed with laboratory tests using an optical tracking system. The results of the pilot study confirm that the robot is fully functional and MRI-compatible in a clinical setting and that it can assist clinicians in complex double-oblique needle insertions.

\section{Materials ANd Methods}

\section{A SYSTEM OVERVIEW}

The robot provides accurate positioning of a needle-guide inside the scanner bore, while the needle insertion is conducted manually outside the bore. This approach was chosen due to the length of the coaxial needle normally used for the ablation procedure (typically $200 \mathrm{~mm}$ ), which would otherwise collide with the magnet bore (GE, Discovery MR750, $3 \mathrm{~T}, 60 \mathrm{~cm}$ nominal bore diameter) and is common to several robotic instruments for MRI-guided percutaneous intervention. ${ }^{17,23,26}$

The main components of the system and their function are depicted in Figure 1. The robot operates in the scanner bore and is secured to the scanner bed. The control unit, which contains the pneumatic valves and the electronics employed to power the robot, is located outside the scanner room in order to minimize the interference to the MRI environment. A set of pneumatic pipes and a shielded encoder cable run through the 115 waveguides and connect the control unit to the robot. A pneumatic multi-connector is used to prevent incorrect piping, while an aluminum waveguide adapter and aluminum connectors are used as interface to the shielded cable. The control unit is supplied by 
medical air (4 bar nominal) and power $(230 \mathrm{~V}, 50 \mathrm{~Hz})$, and communicates with the planning computer via USB2. The planning computer communicates with the robot controller (mbed NXP LPC1768) transmitting the setpoint for the individual cylinders and recording the encoder positions, and receives the MR images from the scanner workstation via Ethernet. On the planning computer, the position and orientation of the needle are computed from the encoder measurements and displayed on the MR images in the graphical user interface. A passive marker (.43 Caliber Clear Paintballs 8000 ,

125 Rap4 UK) mounted on the needle-guide is employed for the robot registration in the MRI scanner using an image processing method based on the convolution theorem of Fourier transforms ${ }^{25}$ Notably, no customization is required on the scanner workstation, which operates with standard imaging sequences.

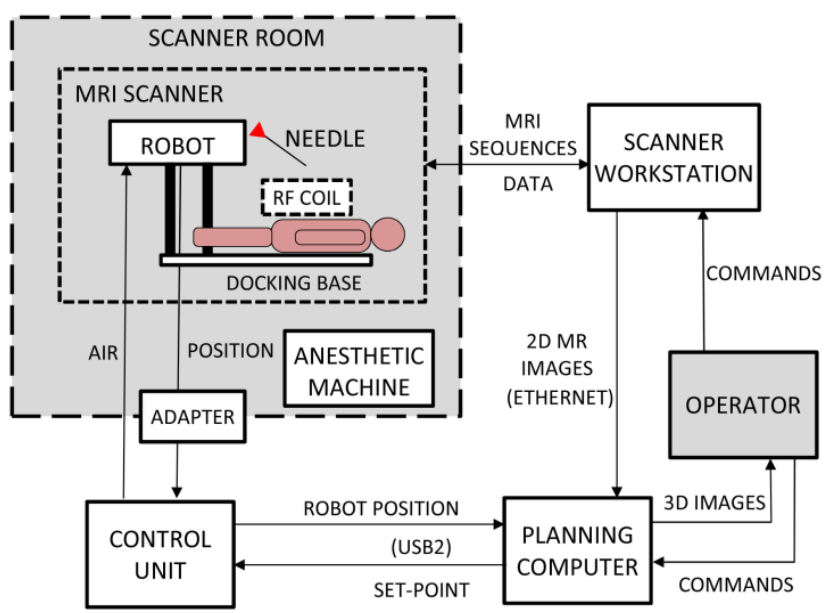

130 Figure 1. Schematic layout for robot-assisted MRI-guided ablation of the liver.

The robot sits on a gantry, manufactured using plastic materials (Delrin, DuPont, Wilmington, USA), and is typically positioned below the patient's waist in order to avoid any contact with the arms. This simplifies the robot setup because padding is not required for the patient's arms and larger patients can be accommodated. A plastic 135 docking base (5 mm thickness) which is positioned on the scanner bed and held in place by the patient's weight, provides a firm and adaptable mounting interface for the gantry. The base is light but rigid, and replicates the profile of the scanner bed, while providing a set of mounting points on both sides of the patient. This solution prevents unwanted movements of the gantry, which could otherwise occur due to the accidental 
140 contact with clinicians or with the RF receiver coils during the setup and that could result in incorrect placement of the needle.
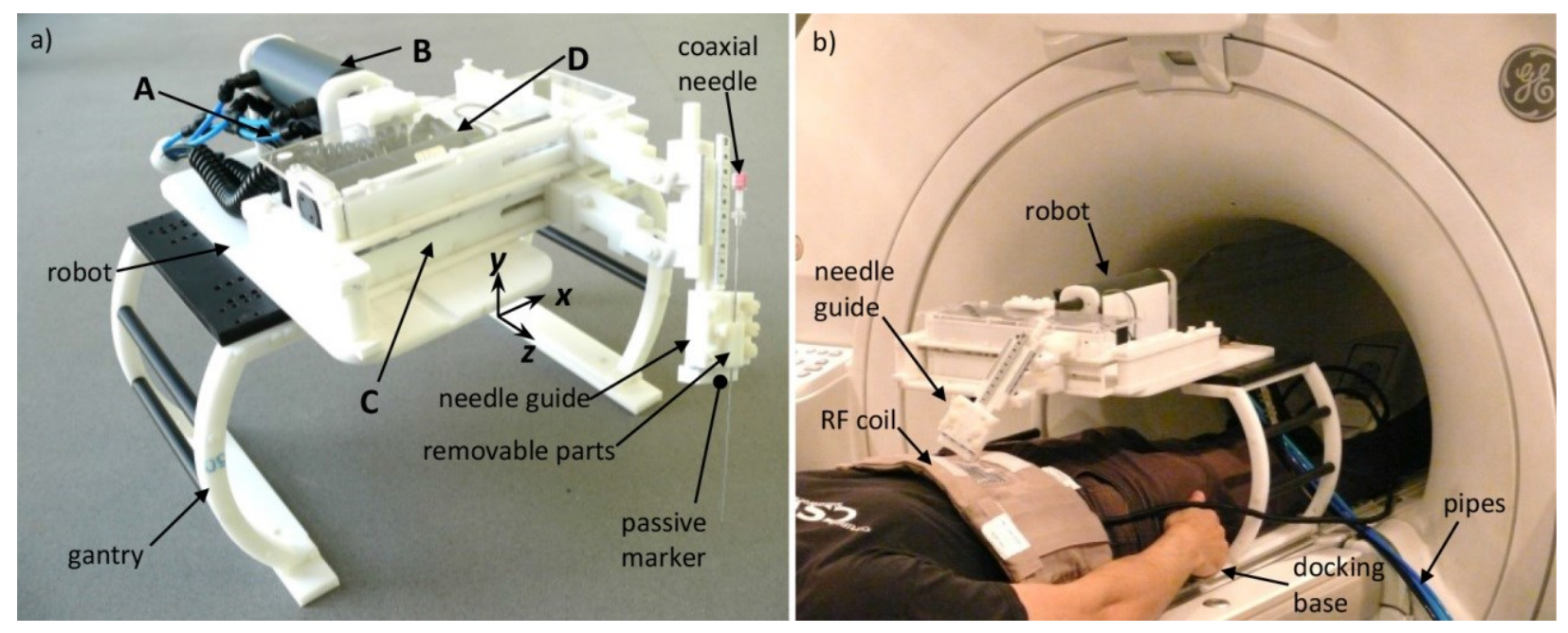

Figure 2. Prototype of the robot (a): the cylinders are $A, B, C, D$; robot setup in the MRI scanner (b). The 3D model of the robot with the docking base is in Figure 4 .

145 The robot is actuated by two pairs of commercially available plastic pneumatic cylinders (IPS Inc., USA) arranged in a hybrid serial-parallel structure that combines high rigidity and large workspace and that provides two orthogonal translations and two inclinations of the needle-guide (Fig. 2). In particular, the needle-guide translates along the axis $\mathbf{Z}$ $(\boldsymbol{x})$ when cylinders $A, B(C, D)$ move together. The differential movements of the 150 cylinders $A, B(C, D)$ results in the inclination of the needle-guide in the $\boldsymbol{y z}(\boldsymbol{x y})$ plane. The axes $(x y z)$ in Figure 2a refer to the robot base frame and are approximately aligned to the coordinate frame of the MRI scanner. Accurate correspondence between robot base frame and MRI coordinate system is established with the robot registration as described in the next section.

\section{B Kinematics}

The following sections present the robot kinematics, which is employed by the control algorithm for the alignment of the needle-guide in MRI-guided percutaneous interventions, and give an overview of the graphical user interface. Since the robot has a hybrid serial-parallel structure, a detailed kinematic analysis is required. 


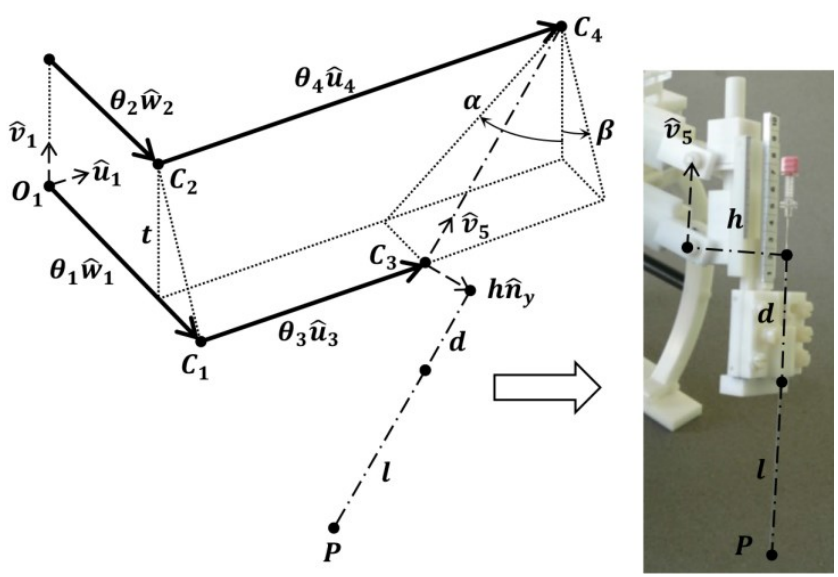

Figure 3. Diagram of the robot kinematics with detail view of the needle-guide.

The direction $\widehat{\boldsymbol{v}}_{\mathbf{5}}$ of the needle axis is dictated by the position $\left(\theta_{1}, \theta_{2}, \theta_{3}, \theta_{4}\right)$ of the four pneumatic cylinders $(A, B, C, D)$ as depicted in Figure 3 . The axes of the cylinders are indicated with the unit vectors $\widehat{\boldsymbol{w}}_{1}, \widehat{\boldsymbol{w}}_{2}, \widehat{\boldsymbol{u}}_{3}, \widehat{\boldsymbol{u}}_{4}$. For construction, $\widehat{\boldsymbol{w}}_{1}$ is parallel to $\widehat{\boldsymbol{w}}_{2}$ and orthogonal to $\widehat{\boldsymbol{u}}_{3}, \widehat{\boldsymbol{u}}_{\mathbf{4}}$. The base frame of the robot is chosen with the axes $\boldsymbol{z}$ and $\boldsymbol{x}$ parallel to $\widehat{\boldsymbol{w}}_{1}$ and $\widehat{\boldsymbol{u}}_{3}$ respectively, the $\boldsymbol{y}$ axis is defined by the vector $\widehat{\boldsymbol{v}}_{\mathbf{1}}=\widehat{\boldsymbol{w}}_{\mathbf{1}} \times \widehat{\boldsymbol{u}}_{3}$, and the origin is $\boldsymbol{O}_{1}$. The cylinders $A$ and $B$ define the position of the points $C_{1}$ and $C_{2}$. The cylinders $C$ and $D$ define the position of the points $C_{3}$ and $C_{4}$ to which the needle-guide is attached with universal joints:

$$
\begin{aligned}
& \boldsymbol{C}_{\mathbf{1}}=\theta_{1} \widehat{\boldsymbol{w}}_{\mathbf{1}} \\
& \boldsymbol{C}_{\mathbf{2}}=\theta_{2} \widehat{\boldsymbol{w}}_{\mathbf{2}}+t \widehat{\boldsymbol{v}}_{\mathbf{1}} \\
& \boldsymbol{C}_{\mathbf{3}}=\boldsymbol{C}_{\mathbf{1}}+\theta_{3} \widehat{\boldsymbol{u}}_{\mathbf{3}} \\
& \boldsymbol{C}_{\mathbf{4}}=\boldsymbol{C}_{\mathbf{2}}+\theta_{4} \widehat{\boldsymbol{u}}_{\mathbf{4}}
\end{aligned}
$$

The term $t$ corresponds to the vertical distance between the points $C_{1}$ and $C_{2}$ which is known from the robot construction (44 mm). The vector $\boldsymbol{C}_{4}-\boldsymbol{C}_{3}=\left\|\boldsymbol{C}_{4} \boldsymbol{C}_{3}\right\| \hat{\boldsymbol{v}}_{5}$ is parallel to the needle axis for construction. The position $\boldsymbol{P}$ of the needle tip in the robot base frame is given by:

$$
\boldsymbol{P}=\boldsymbol{C}_{3}-(l+d) \widehat{\boldsymbol{v}}_{5}+h\left(\left(\widehat{\boldsymbol{v}}_{5} \times \widehat{\boldsymbol{w}}_{1}\right) \times \widehat{\boldsymbol{v}}_{5}\right)
$$


175 The term $h$, known from the needle-guide construction $(38 \mathrm{~mm})$, is the distance between the needle axis and the vector $\boldsymbol{C}_{4}-\boldsymbol{C}_{3}$. The term $d(22.5 \mathrm{~mm})$ is the length of the needle-guide measured from $C_{3}$, while $l$ is the length of the portion of the needle extending out of the needle guide $(l \leq 200 \mathrm{~mm}$; typical needle length $=200 \mathrm{~mm})$. The inclination of the needle axis is described by the angles $\alpha, \beta$ which are defined by the projections of the vector $\boldsymbol{C}_{4}-\boldsymbol{C}_{3}$ on the planes $\boldsymbol{x y}$ and $\boldsymbol{y z}$ :

$$
\begin{aligned}
& \beta=\tan ^{-1}\left(\frac{\theta_{2}-\theta_{1}}{t}\right)=\cos ^{-1}\left(\frac{t}{\left\|\boldsymbol{C}_{2} \boldsymbol{C}_{\mathbf{1}}\right\|}\right) \\
& \alpha=\tan ^{-1}\left(\frac{\theta_{4}-\theta_{3}}{t}\right)=\cos ^{-1}\left(\frac{t}{\left\|\left(\boldsymbol{C}_{\mathbf{4}}-\boldsymbol{C}_{3}\right)-t \tan (\beta) \widehat{\boldsymbol{w}}_{\mathbf{1}}\right\|}\right)
\end{aligned}
$$

The transformation that relates the robot base frame to the coordinate system of the MRI scanner is computed during the registration process. Firstly, the position of the passive marker mounted on the needle-guide is calculated from the MR images when the pistons are retracted and $\alpha, \beta=0$. Subsequently, the position of the origin $\boldsymbol{O}_{\mathbf{1}}$ is calculated knowing the values of $d, h$ from the robot construction. The needle-guide is then moved to 2 different points $\left(\theta_{1}=40 ; \theta_{3}=40\right)$ and the position of the marker is calculated again. This procedure defines 3 points that uniquely identify the transformation. ${ }^{25}$ Finally, the position of the needle tip calculated with (2) for a given length $l$ is expressed in the coordinate system of the MRI scanner $\left(\boldsymbol{P}_{M_{R}}\right)$ and displayed on the graphical user interface.

The reachable workspace is calculated evaluating (1) and (2) for all admitted positions $\left(\theta_{1}, \theta_{2}, \theta_{3}, \theta_{4}\right)$ and for $l \leq 200 \mathrm{~mm}$. For simulation purposes the cylinder positions were incremented from 0 to the maximum (either $90 \mathrm{~mm}$ or $145 \mathrm{~mm}$ ) in steps of $5 \mathrm{~mm}$ (Fig. 4). For construction, the distance $\left\|C_{4} C_{3}\right\|$ should remain smaller than $100 \mathrm{~mm}$, 195 corresponding to the travel of the sliding mechanism in the needle-guide which is limited by the space constraints in the MRI scanner. For simplicity, the following limits are therefore imposed by the controller on the angles:

$$
-50^{\circ}<\alpha<50^{\circ} ;-60^{\circ}<\beta<25^{\circ} ;
$$

In summary, the robot has 4 degrees-of-freedom (DOF): two translations $\theta_{1}, \theta_{3}$ and two inclinations $\alpha, \beta$. The translational range of motion of the needle-guide is $90 \mathrm{~mm}$ for $\theta_{3}$, 
$200145 \mathrm{~mm}$ for $\theta_{1}$, while the full angular range of motion is $100^{\circ}$ for $\alpha$ and $85^{\circ}$ for $\beta$. Simulations on an average-size adult model ${ }^{28}$ indicate that the robot-assisted needle insertion can reach more than $95 \%$ of the liver with a $200 \mathrm{~mm}$ long needle.

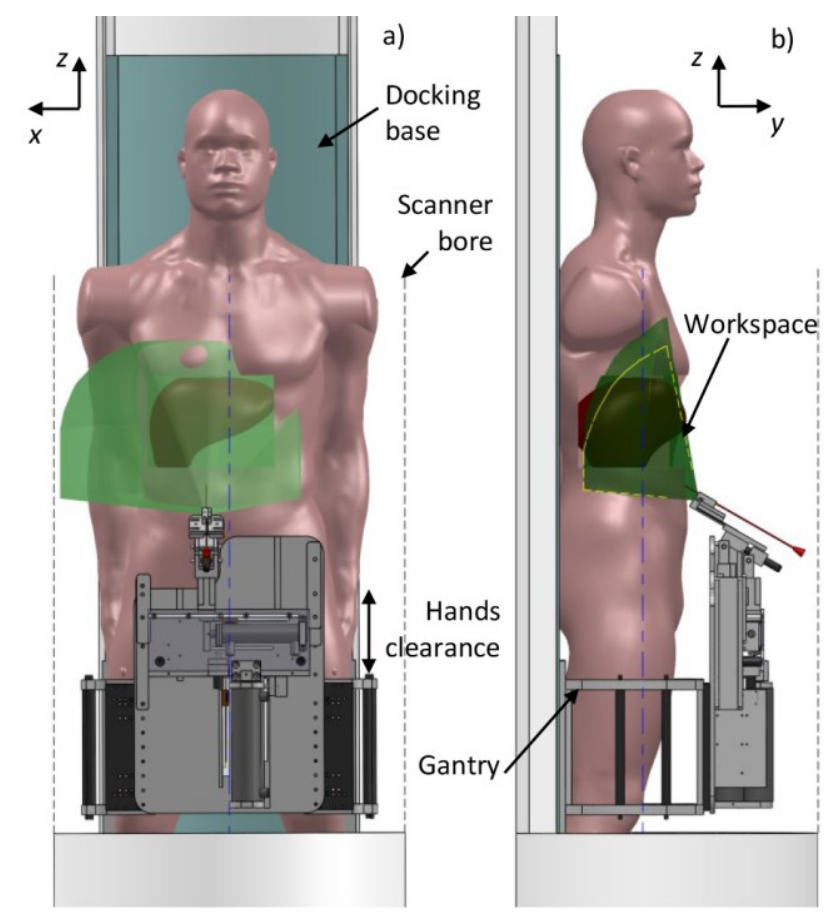

Figure 4. Intersection between the robot workspace for $\beta<0^{\circ}$ (green) and the liver 205 model (red): top view (a); side view (b). Dashed yellow line represents the workspace of our previous prototype. ${ }^{25}$

\section{B.2 Inverse Kinematics}

Given the target point $\boldsymbol{P}^{\prime}$ and the desired needle insertion direction $\widehat{\boldsymbol{v}}_{\mathbf{5}}$ expressed in the robot base frame, inverse kinematics equations compute the corresponding position of 210 the four pneumatic cylinders $\left(\theta_{1}, \theta_{2}, \theta_{3}, \theta_{4}\right)$. Substituting (1) into (2) and inverting (3), the position of the cylinders is computed as: 


$$
\begin{aligned}
\theta_{1} & =\boldsymbol{P}^{\prime} \cdot \widehat{\boldsymbol{w}}_{\mathbf{1}}+(l+d) \widehat{\boldsymbol{v}}_{\mathbf{5}} \cdot \widehat{\boldsymbol{w}}_{\mathbf{1}}-h\left(\left(\widehat{\boldsymbol{v}}_{\mathbf{5}} \times \widehat{\boldsymbol{w}}_{\mathbf{1}}\right) \times \widehat{\boldsymbol{v}}_{\mathbf{5}}\right) \cdot \widehat{\boldsymbol{w}}_{\mathbf{1}} \\
\theta_{2} & =\theta_{1}+\tan (\beta) t \\
\theta_{3} & =\boldsymbol{P}^{\prime} \cdot \widehat{\boldsymbol{u}}_{\mathbf{3}}+(l+d) \widehat{\boldsymbol{v}}_{\mathbf{5}} \cdot \widehat{\boldsymbol{u}}_{\mathbf{3}}-h\left(\left(\widehat{\boldsymbol{v}}_{\mathbf{5}} \times \widehat{\boldsymbol{w}}_{\mathbf{1}}\right) \times \widehat{\boldsymbol{v}}_{\mathbf{5}}\right) \cdot \widehat{\boldsymbol{u}}_{\mathbf{3}} \\
\theta_{4} & =\theta_{3}+\tan (\alpha) t \\
l & =\frac{h\left(\left(\widehat{\boldsymbol{v}}_{\mathbf{5}} \times \widehat{\boldsymbol{w}}_{\mathbf{1}}\right) \times \widehat{\boldsymbol{v}}_{\mathbf{5}}\right) \cdot \widehat{\boldsymbol{v}}_{\mathbf{1}}-\boldsymbol{P}^{\prime} \cdot \widehat{\boldsymbol{v}}_{\mathbf{1}}}{\widehat{\boldsymbol{v}}_{\mathbf{5}} \cdot \widehat{\boldsymbol{v}}_{\mathbf{1}}}-d
\end{aligned}
$$

The needle insertion direction $\widehat{v}_{\mathbf{5}}$ is defined by the angles $\alpha, \beta$ which are specified by the clinicians based on the following considerations: 1) needle length; 2) minimum insertion depth required for the laser ablation, which is related to the active diffusion fiber length;

215 3) position of the gall bladder; 4) position of major blood vessels; 5) position of the ribs; 6 ) space constraints of the scanner bore. In practice, choosing the target point $\boldsymbol{P}^{\prime}$ and the direction $\widehat{\boldsymbol{v}}_{5}$ might require a few iterations depending on the size and location of the lesion and on the anatomy of the patient (e.g. position of the liver and of the gall bladder relative to the rib cage). To this end, different imaging sequences are employed in order to provide sufficient information to the clinicians.

\section{B.3 Graphical user interface}
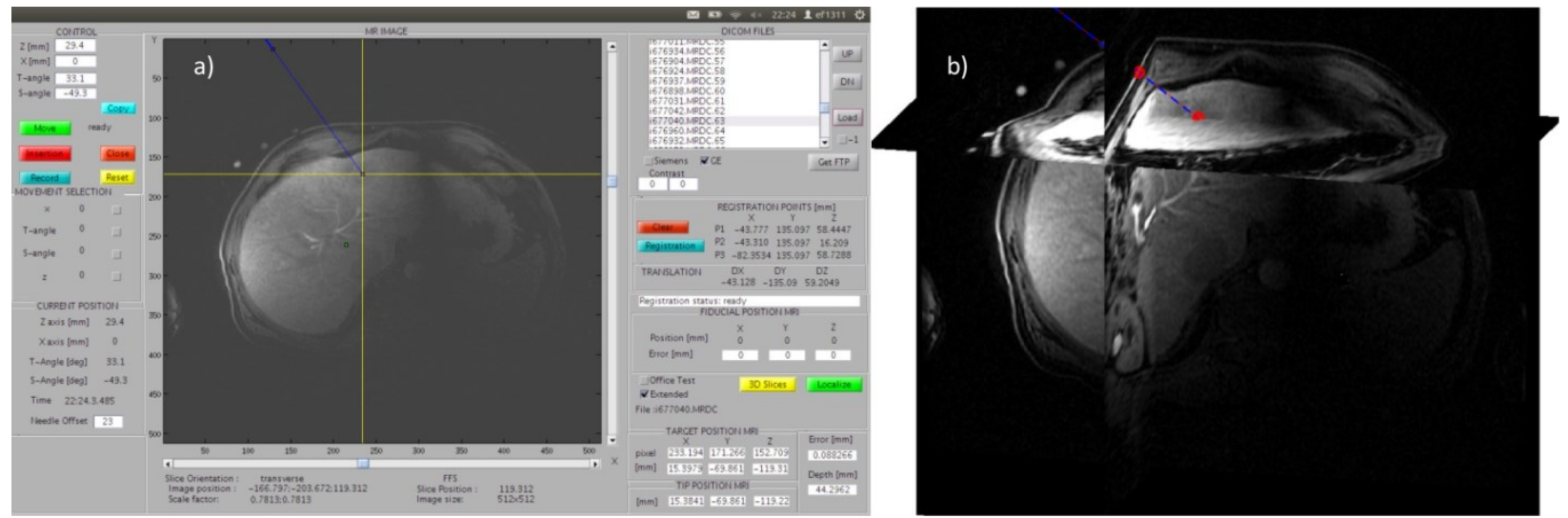

Figure 5. Graphical user interface: slice view (a): the crosshair identifies the lesion; volume view (b): the bottom red sphere is positioned on the target lesion, while the top one is at the incision site. The needle axis in both images is plotted in blue. The images refer to Patient 2 in the pilot study.

The current version of the graphical user interface is designed to accept MR images in the axial orientation. A series of slices is usually acquired under breath-hold ${ }^{29}$ with a 
clinically relevant imaging sequence (e.g. Ultra fast Gradient Echo) and transferred to 230 the planning computer. The clinicians browse through the slices, which are ordered along the longitudinal $(z)$ axis of the MRI scanner, until the tumor becomes visible. The tumor is then selected moving a crosshair on the chosen slice (Fig. 5a). The slices can also be displayed collectively as a volume, together with the target position, the needle tip position, and the needle axis. A cursor can be dragged along the needle axis to

235 calculate the insertion depth. The volume can also be rotated and sectioned with three orthogonal planes for visualization purposes (Fig. 5b).

\section{C RoBOt CONTROL}

The four pneumatic cylinders are powered by proportional pressure regulators (Tecno Basic, Hoerbiger, Germany) that control their output pressure in closed loop with the onboard electronics. The control voltage is supplied to the proportional valves by 12-bit digital-to-analog converters (DAC). Four linear encoders (EM1-250, US Digital, Vancouver, WA, USA) are employed together with quadrature counters to measure the pistons position with $25 \mu \mathrm{m}$ resolution. The DAC and the quadrature counters communicate with the microcontroller via a serial-peripheral-interface (SPI) bus at 400

$245 \mathrm{kHz}$. For the individual cylinders, positioning accuracy comparable to the encoder resolution $(30 \mu \mathrm{m})$ was achieved with TDC algorithms. ${ }^{25} \mathrm{~A}$ high-level control strategy is employed to facilitate needle-guide alignment and is outlined in Figure 6.

The inverse kinematics is initially solved for the specified target point $\boldsymbol{P}^{\prime}$ and the insertion direction $\widehat{v}_{5}$. In case $\alpha, \beta$ are outside the range (4), their limit values are fed

250 back into the inverse kinematics (5) which computes new values of $\theta_{1}, \theta_{2}, \theta_{3}, \theta_{4}, l$. The ability to automatically adjust the angles $\alpha, \beta$ is also provided as an additional feature in the user interface: in case the positions $\theta_{3}, \theta_{1}$ reach their limit $(90 \mathrm{~mm}$ or $145 \mathrm{~mm})$, the angles $\alpha, \beta$ are computed recursively from the forward kinematics (2-3). In particular, $\theta_{4}, \theta_{2}$ are either incremented or decremented by one encoder step at each iteration until 255 the following condition is met: $\left\|\boldsymbol{P}^{\prime}-\boldsymbol{P}\right\| \leq 0.1 \mathrm{~mm}$.

The calculated setpoint is then communicated to the robot controller that initiates the motion. After the movement is completed, the encoder counters are automatically 
disabled and the proportional regulators are reset to the rest pressures. New MR images can then be acquired avoiding motion artefacts, ${ }^{14}$ while the position measurements are not affected by the RF noise. The forward kinematics (2) is updated after each movement and the user interface displays the calculated needle position on the planning computer for verification purposes. The clinicians assess whether the computed insertion direction and the needle tip position are appropriate, in which case the cylinder chambers are pressurized in order to increase the rigidity of the robot 265 during the manual needle insertion. Otherwise, a different target point or a different insertion direction are communicated to the controller.

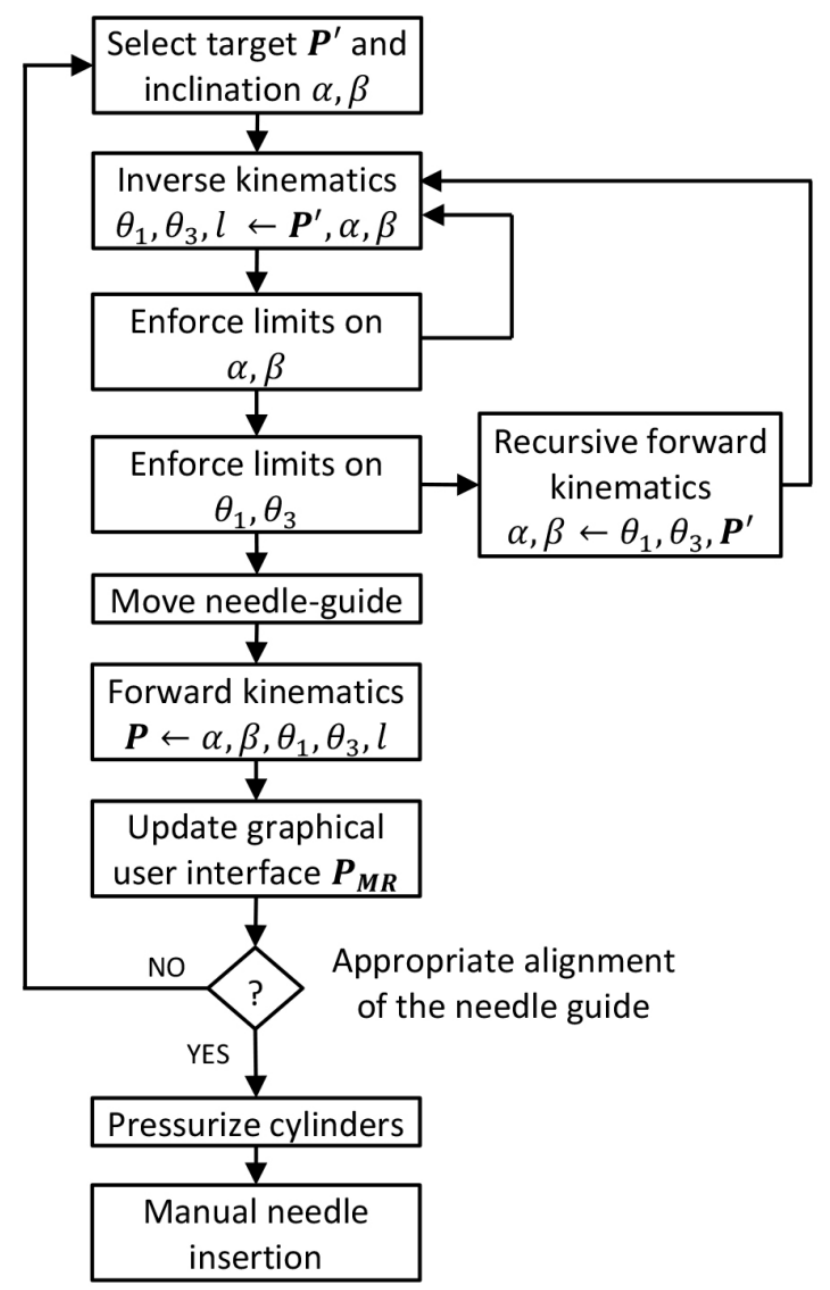

Figure 6. Robot control algorithm.

\section{D Clinical Workflow}


270 A clinical feasibility assessment was initially conducted with a healthy volunteer subject (male, age 36, weight $75 \mathrm{~kg}$ ) in preparation for the clinical use of the robot (Fig. 2b). The workflow for the MRI-guided liver ablation was then defined in close collaboration with the clinicians. The main phases of the workflow are summarized in Figure 7.

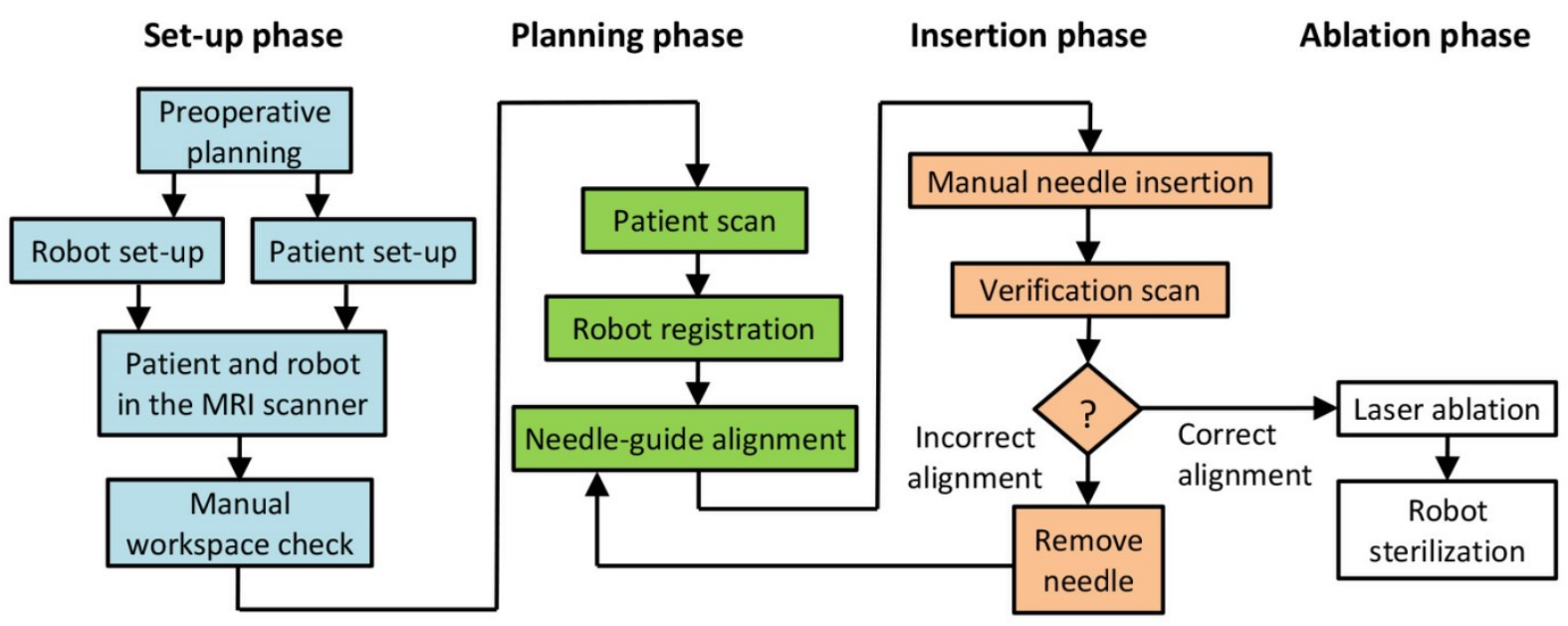

275 Figure 7. Workflow of the robot-assisted MRI-guided ablation of the liver.

Initially, the clinicians assess the preoperative MR images to locate the lesion and start considering appropriate insertion directions. The robot is transported to the scanner room and the needle-guide, previously sterilized, is mounted on the actuators using sterile gloves. As part of the setup, the robot is connected to the control unit with 280 pneumatic pipes and with the shielded cable but it is not powered on at this stage. The planning computer is connected to the control unit and to the scanner workstation (Fig. 1). These operations are completed while the patient is being prepared for the procedure outside the scanner room and treated with general anesthetic. The docking base is then positioned on the scanner bed. The patient is moved in the scanner room and placed on the scanner bed in the supine position. A flexible RF receiver array coil (4 channels, GP Flex, GE) is secured above the patient's abdomen with straps. The gantry is attached to the docking base and the robot is mounted on the gantry. The needleguide can then be moved manually to confirm that the approximate location of the lesion is within the workspace. If this is not the case, the robot can be attached to the gantry in a different position. Finally, the scanner bed is moved inside the bore and the clinicians, 
apart from the anesthetist who closely monitors the patient throughout the procedure, leave the scanner room.

Initial MR images are acquired during breath-hold to locate the lesion (e.g. Axial Ultra fast Gradient Echo, Repetition Time $=4.13 \mathrm{~ms}$, Echo Time $=1.88 \mathrm{~ms}$, Flip Angle $=12^{\circ}$, Field of View $=400 \mathrm{~mm} \times 400 \mathrm{~mm}$, slice thickness $=4 \mathrm{~mm}$, slice number $=90$ ). Notably, the patient lies under general anesthesia and is ventilated and closely monitored by the anesthetist throughout the procedure. Breath-hold is employed in order to avoid motion artefacts, as in conventional freehand MRI-guided percutaneous interventions. ${ }^{29}$ Subsequently, the robot is powered on, the registration program is launched on the planning computer, and a new set of MR images is acquired (e.g. Ultra fast Gradient Echo, Repetition Time $=34 \mathrm{~ms}$, Echo Time $=3.8 \mathrm{~ms}$, Flip Angle $=20^{\circ}$, Field of View $=$ $400 \mathrm{~mm} \times 400 \mathrm{~mm}$, slice thickness $=2 \mathrm{~mm}$, slice number =6). For the registration, the slice position is preset on the scanner workstation according to the height of the needleguide above the scanner bed, which is known from the robot construction. This approach reduces the registration time, which is additional to the conventional freehand procedure. The planning computer calculates the position of the passive marker mounted on the needle-guide (ref. Fig. 2) in three different positions and computes the transformation between the robot base frame and the scanner coordinate frame (Ref. Section 2.B). The registration is only required once per procedure and typically takes less than 5 minutes, however it can be repeated in case the robot or the gantry need to be repositioned. The planning software is then launched and the initial MR images are visualized. The clinicians specify the target point and the insertion direction on the graphical user interface. The robot moves the needle-guide accordingly and the user interface displays the computed position of the needle axis and calculates the insertion depth (ref. Section 2.C).

At this point, the clinicians move to the scanner room for the manual needle insertion and the scanner bed slides out of the bore. The RF receiver coil is temporarily removed to allow access to the incision site, which is disinfected. The clinicians palpate the patient's abdomen to confirm the location of the ribs which might not be clearly visible on the MR images. The coaxial needle (Somatex, Germany) is inserted in the needle- 
guide and is then pushed manually by the clinicians to the prescribed depth, which is measured with the depth gauge marked on needle. The clinicians can release the needle from the needle-guide at any time leaving it free to move as a result of patient's respiration. Subsequently, the RF receiver coil is positioned on the patient's abdomen

325 while the needle is left in place. The scanner bed is moved back into the bore, and a new set of MR images is acquired under breath-hold for verification purposes. If the needle artifact that appears on the MR images intersects with the lesion and its position is considered appropriate, the clinicians prepare for the ablation therapy. Otherwise, the needle is removed and the insertion is repeated.

330 The ablation therapy employs a laser applicator and a laser source (continuous wave, $\mathrm{Nd}$ :YAG, $1064 \mathrm{~nm}$ ) to heat the lesion. ${ }^{2}$ The coaxial needle is replaced with the laser applicator using a titanium guide wire and the ablation therapy is administered. Further MR images are acquired throughout the ablation for the purpose of thermal monitoring. Subsequently, the laser applicator is removed and the wound is cleaned. Finally, the robot is removed from the scanner room and the needle-guide is disassembled for sterilization.

\section{E SAFETY AND SteriLizATION}

The safe operation of the robot is ensured by the robot design and by the verification checkpoints introduced in the control algorithm and in the workflow. All sharp edges in

340 the gantry and on the robot structure were chamfered. The robot is set up in the MRI scanner under the supervision of the clinicians, who decide the most appropriate location of the gantry and of the actuated unit considering the size of the patient and the position of the RF receiver coil. Additionally, the needle-guide can be moved manually during the setup to ensure that no contact with the patient or the RF receiver coil can

occur. Before attempting the needle insertion, the position of the needle-guide is verified on the planning computer, which also calculates the expected position error of the needle tip. The air supply and the power supply can be isolated pressing the stop button on the control box and the robot can be removed at any time from the scanner bed.

The needle-guide is disassembled after each procedure and its removable parts, which 
350 are the only components coming in direct contact with the coaxial needle, can be either sterilized or disposed of. The gantry and the robot structure are cleaned with sterile towels and disinfectant solution. The needle-guide is assembled again before the new procedure using sterile gloves.

\section{F LABORATORY TESTING}

355 The position repeatability of the robot was assessed with an optical tracking system (Optotrak, NDI, Canada, $0.1 \mathrm{~mm}$ nominal accuracy). An optical marker (Smart Marker, $\mathrm{NDI}$, Canada) was attached to the needle-guide in correspondence with the passive marker (ref. Fig. 2a) and the robot was programmed to approach a reference point 10 times from a random position in the workspace. The coordinates of the maker were

360 measured with the tracking system every time the robot stopped at the reference point.

The robot accuracy was assessed mounting an optical marker on an adapter (195 mm long) attached to the needle-guide in order to simulate the position of the needle tip. Initially, the transformation between the robot base frame and the coordinate frame of the tracking system was computed. This entailed moving the needle-guide to 3 different points and measuring the corresponding marker position with the tracking system. Subsequently, the measurements from the tracking system (200 for each point) were expressed in the robot base frame. The maker position at 25 different points was then compared to the values computed with the forward kinematics.

\section{G Preliminary Patient StUdy}

Robot-assisted MRI-guided laser ablations of the liver were conducted at St Mary's Hospital, London, UK, by an experienced interventional radiologist and his group, with technical support provided by the research team. The aim of the study was to confirm that the robot is fully functional in a clinical setting and that it can be used for MRIguided percutaneous intervention. The procedure was approved by the institutional 375 research ethics committee, and written informed consent was given by the patients prior to all test sessions. Three patients enrolled in this initial study (Table 1) and the robot was used to assist the first needle insertion attempt in two of them, while any follow up was done manually, as in freehand MRI-guided procedures. ${ }^{29}$ The procedure followed 
the workflow outlined in Section 2.D.

\section{Results}

\section{A LABORATORY TESTING}

The robot repeatability was assessed at an arbitrary reference point within the workspace $\left(\theta_{1}=51 \mathrm{~mm} ; \theta_{3}=71 \mathrm{~mm} ; \alpha=-30^{\circ} ; \beta=-5^{\circ}\right)$. The robot approached the references point 10 times (200 measurements for each point, 2000 in total). The mean error was $0.35 \mathrm{~mm}$ with a standard deviation of $0.32 \mathrm{~mm}$. The robot accuracy was assessed for 25 different fixed points distributed within the workspace. The test was repeated twice and the mean error was $2.5 \mathrm{~mm}$ with a standard deviation of $1.2 \mathrm{~mm}$.

In our previous work, the MRI-compatibility of the system was assessed in a $3 T$ MRI scanner (Siemens Verio) with the robot positioned in the scanner bore and connected to 390 the control unit. The variation in image SNR computed according to ${ }^{30}$ for clinically relevant imaging sequences remained below $5 \%$ for all test conditions, including with the needle-guide in motion, and no visible image artifacts were detected. ${ }^{25}$ Additionally, no effects on the encoder signals were observed as a result of the MRI environment. Finally, the needle placement error under MRI guidance was assessed in experiments 395 with a gelatin phantom using cod-liver-oil capsules (Boots, UK) as targets. The mean position error of the needle tip over 10 needle insertions, computed from the MR images, was $2.9 \mathrm{~mm}$ with a standard deviation of $1.4 \mathrm{~mm} .^{25}$
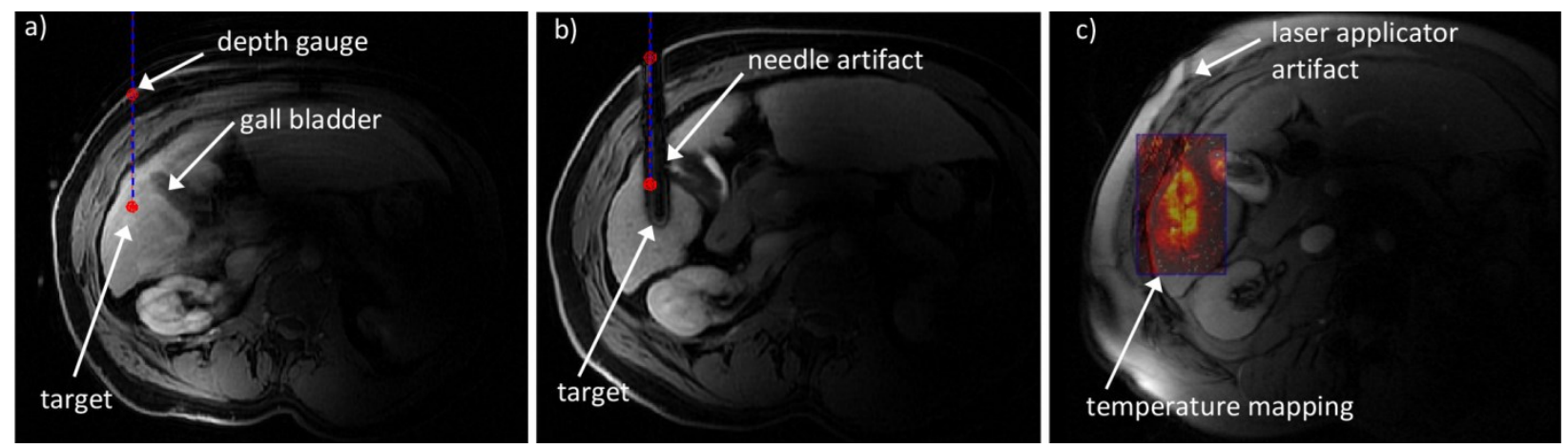

Figure 8. MR images for Patient 1: planning of the needle insertion (a); verification image (b); temperature mapping (c). 


\section{B Preliminary patient Study}

For Patient 1 , an insertion direction parallel to the $y$ axis was chosen by the clinicians as the most suitable (Fig. 8a). The robot setup took approximately 30 minutes, while the robot registration was competed in less than 4 minutes. The planning phase and the insertion phase (ref. Fig. 7) took 37 minutes, including the scan time. The position error at the needle tip, computed with the forward kinematics (2), was less than $0.1 \mathrm{~mm}$ (ref. Section 2.B). However, the needle artifact on the verification MR images showed that the needle axis was deflected and the insertion was deeper than required (Fig. 8b). Consequently, the needle position was corrected manually in a second insertion 410 attempt.

Table 1. Pilot study on patients.

\begin{tabular}{|c|c|c|c|}
\hline & Patient 1 & Patient 2 & Patient 3 \\
\hline Age (years) & 60 & 60 & 70 \\
\hline $\operatorname{Sex}(M / F)$ & M & M & $\mathrm{F}$ \\
\hline Weight $(\mathrm{kg})$ & 65.0 & 54.4 & 79 \\
\hline Lesion diameter (mm) & 10 & 16 & 22 \\
\hline$\theta_{1}(\mathrm{~mm})$ & 44.3 & 29.4 & 117.8 \\
\hline$\theta_{3}(\mathrm{~mm})$ & 58.8 & 0.0 & 59.7 \\
\hline$\alpha\left(^{\circ}\right)$ & 0.0 & 33.1 & 9.6 \\
\hline$\beta\left(^{\circ}\right)$ & 0.0 & -49.3 & -9.5 \\
\hline Insertion depth (mm) & 95 & 65 & 230 \\
\hline Computed position error (mm) & 0.08 & 0.03 & - \\
\hline Robot-assisted insertions & 1 & 1 & - \\
\hline Total needle insertions ${ }^{1}$ & 2 & 4 & 8 \\
\hline Needle insertion duration $(\min )^{2}$ & 37 & 44 & - \\
\hline
\end{tabular}

${ }^{1}$ Only the first attempt is robot-assisted.

${ }^{2}$ Refers to the first needle insertion and includes robot registration, planning, robotassisted needle insertion, and verification.

For Patient 2, a double oblique insertion direction was chosen in order not to puncture 415 the gall bladder and to achieve a sufficient insertion depth (ref. Section 2.B). In this 
case, the target lesion was correctly punctured at the first insertion attempt. No visible needle deflection or tissue shifts, that are more common for deeper insertions, were observed on the verification images. Due to the larger size of the lesion, a second needle was inserted manually next to the first in order to accommodate two laser

420 applicators and to treat a larger area. Notably, the freehand insertion required three attempts.

Patient 3 was considerably heavier compared to the others, and the insertion depth was larger than in previously recorded robot-assisted MRI-guided percutaneous interventions. ${ }^{27}$ The procedure was attempted by the clinicians in order to assess the 425 potential issues arising with heavier patients. Notably, in this case the clinicians could not palpate the rib cage due to the patient's size and the needle could not be inserted completely at the first attempt because of the contact with a rib. In total eight freehand insertion attempts were required to correctly target the lesion. This result is in agreement with the retrospective study presented in ${ }^{6}$ that indicated an average of five insertion attempts for MRI-guided liver biopsies.

The results of the pilot study are summarized in Table 1. The lesion size and, for the first two patients, the insertion depth are within the range defined in previous clinical studies. ${ }^{5}$ Notably, the presence of the robot did not visibly affect the MR images. Finally, the laser ablation procedure was successfully completed in all three cases without complications.

\section{Discussion}

This paper presented a robotic instrument for MRI-guided ablation of the liver describing the design, the control algorithm and the user interface, and the clinical workflow. Differently from other systems, ${ }^{8,} 26$ the robot was designed to operate in closed-bore

$440 \mathrm{MRI}$ scanners and can realign to different incision sites without manual intervention. Additionally, the robot is actuated by commercially available pneumatic cylinders resulting in affordability and lower system complexity. The repeatability and position accuracy of the robot were assessed in a laboratory environment using an optical tracking system. The results confirm that the accuracy is appropriate for liver tumors 
445 normally treated with laser ablation, which have a diameter from $10 \mathrm{~mm}$ to $50 \mathrm{~mm} .^{2}$

The robot was subsequently employed in an initial pilot study on patients. The robot did not interfere with the equipment used for MRI-guided laser ablation and did not visibly affect the MR images, confirming the results of our previous MRI-compatibility tests on phantoms. ${ }^{25}$ The robot setup was completed during the time required to prepare the 450 patient, while the robot registration took only 4 minutes and was only conducted once at the start of each procedure. The time required for the robot-assisted needle insertion was larger than that recorded during phantom trials $(21 \text { minutes })^{25}$ due to the higher anatomic complexity of the patients which required prolonged scan time. Notably, only conventional MR imaging sequences were employed and no specific training was 455 required for the clinicians. This is in contrast with existing systems that require a close integration with the MRI scanner. ${ }^{23}$ The robot-assisted needle insertion was successfully completed on two patients, one of which required a double oblique insertion. Previous clinical studies ${ }^{5,27}$ suggested that double oblique insertions are particularly difficult to map mentally and can therefore require several attempts. The discrepancy between

460 calculated and actual needle tip position was attributed to needle bending due to tissue inhomogeneity, and to tissue shifts due to organ movement. Finally, the erroneous insertion depth, which was manually set by the clinicians, was ascribed to the low resolution of the depth gauge on the coaxial needle $(10 \mathrm{~mm})$. The results confirm that the robot can be used in a clinical setting in its current form. Further examination is required in order to assess the clinical benefits of robot-assisted percutaneous interventions. The study also highlighted the difficulties related to heavier patients and the challenges associated with targeting deeper lesions. In particular, targeting deeper lesions could require more insertion attempts due to the higher chance of needle deflection. Additionally, palpating the rib cage in obese patients might be more difficult

470 for clinicians. Also, with obese patients it might not be possible to accommodate the RF receiver coil and the robot inside the MRI scanner bore due to the space constraints. In this respect, careful patient selection appears essential for the effective use of the robot.

The main limitation of the system that emerged as a result of this study is due to the space constraints of the MRI scanner which posed restrictions on the choice of the RF 
475 receiver coil. However, while the pilot study was conducted in a scanner with a relatively small bore size, the robot was designed to also operate in wide-bore scanners. This could allow using larger and more sophisticated RF receiver coils that might further enhance the accuracy of the system. A further limitation was the low resolution of the depth gauge on the coaxial needle which was used to measure the insertion depth. As part of the future work, a more accurate way of measuring the insertion depth will be devised. Finally, a current limitation of the graphical user interface is the ability to acquire MR images only in the axial orientation. Future work will address this point and will also assess the possibility of fusing images acquired with different scanning sequences in order to better visualize the patient's anatomy. Additionally, the 3D

485 visualization of the scanned volume could be enhanced in order to further improve the planning phase and make it more intuitive for clinicians. In this respect, providing different and more intuitive ways of specifying the target location and the needle entry point on the scanned volume could further reduce the number of insertion attempts. While employing breath-hold as in conventional freehand needle insertions minimizes tissue shift due to respiration, ${ }^{29}$ more advanced strategies for motion compensation could be investigated. Finally, an extended study on a larger number of patients will be conducted to quantify the benefits of using the robot in MRI-guided ablations of the liver. Furthermore we intend to explore the use of the robot also for other ablation modalities, such as microwave ablation.

\section{Conclusion}

In conclusion we have demonstrated that the proposed robotic instrument is fully functional in a clinical setting and that it can assist clinicians in MRI-guided laser ablations of liver tumors. Additionally, it could be employed for MRI-guided biopsies in the abdominal region, and it could serve as training tool for less experienced clinicians. 500 Further examination is required in order to assess the clinical benefits of robot-assisted percutaneous interventions under MRI-guidance. 


\section{ACKNOWLEDGMENTS}

The authors acknowledge the funding from the National Institute for Health Research through the i4i Grant II-AR-1109-11011.

\section{CONFLICT OF INTEREST}

The authors have no relevant conflicts of interest to disclose.

\section{References}

1 T.J. Vogl, P.K. Müller, R. Hammerstingl, N. Weinhold, M.G. Mack, C. Philipp, M. Deimling, J. Beuthan, W. Pegios, and H. Riess, "Malignant liver tumors treated 510 with MR imaging-guided laser-induced thermotherapy: technique and prospective results," Radiology 196(1), 257-265 (1995).

2 A.L. Gough-Palmer and W.M.W. Gedroyc, "Laser ablation of hepatocellular carcinoma-A review," World J. Gastroenterol. 14(47), 7170-7174 (2008).

3 W.M.W. Gedroyc, "Magnetic resonance guidance of thermal ablation," Top. Magn. Reson. Imaging 16(5), 339-353 (2005).

4 E.M. Kardoulaki, R.R.A. Syms, I.R. Young, K. Choonee, M. Rea, and W.M.W. Gedroyc, "Optothermal profile of an ablation catheter with integrated microcoil for MR-thermometry during Nd:YAG laser interstitial thermal therapies of the liveran in-vitro experimental and theoretical study," Med. Phys. 42(3), 1389-97 (2015). M. Moche, S. Heinig, N. Garnov, J. Fuchs, T.-O. Petersen, D. Seider, P. Brandmaier, T. Kahn, and $\mathrm{H}$. Busse, "Navigated MRI-guided liver biopsies in a closed-bore scanner: experience in 52 patients," Eur. Radiol. (Epub ahead of print), 1-9 (2015). evaluation of a double ring RCM mechanism for robotic needle guidance in MRIguided liver interventions," in 2013 IEEE/RSJ Int. Conf. Intell. Robot. Syst.(IEEE, 2013), pp. 4078-4083.

7 C.M. Pacella, G. Francica, and G.G. Di Costanzo, "Laser Ablation for Small Hepatocellular Carcinoma," Radiol. Res. Pract. 2011, 1-8 (2011). N. Hata, J. Tokuda, S. Hurwitz, and S. Morikawa, "MRI-compatible manipulator with remote-center-of-motion control," J Magn Reson Imaging 27(5), 1130-1138 (2008).

$9 \quad$ N. Mylonas and C. Damianou, "MR compatible positioning device for guiding a focused ultrasound system for the treatment of brain deseases," Int. J. Med. Robot. 10(1), 1-10 (2014). 
E. Epaminonda, T. Drakos, C. Kalogirou, M. Theodoulou, C. Yiallouras, and C. Damianou, "MRI guided focused ultrasound robotic system for the treatment of gynaecological tumors," Int. J. Med. Robot. 12(1), 46-52 (2015).

S. Eslami, W. Shang, G. Li, N. Patel, G.S. Fischer, J. Tokuda, N. Hata, C.M. Tempany, and I. Iordachita, "In-bore prostate transperineal interventions with an MRI-guided parallel manipulator: system development and preliminary evaluation," Int. J. Med. Robot. (Epub ahead of print), 1-15 (2015).

H. Su, W. Shang, G. Cole, G. Li, K. Harrington, A. Camilo, J. Tokuda, C.M. Tempany, N. Hata, and G.S. Fischer, "Piezoelectrically Actuated Robotic System for MRI-Guided Prostate Percutaneous Therapy," IEEE ASME Trans. Mechatron. 20(4), 1920-1932 (2015).

M.A. Tavallaei, P.M. Johnson, J. Liu, and M. Drangova, "Design and evaluation of an MRI-compatible linear motion stage," Med. Phys. 43(1), 62-71 (2016).

A. Krieger, S.-E. Song, N.B. Cho, I. Iordachita, P. Guion, G. Fichtinger, and L.L. Whitcomb, "Development and Evaluation of an Actuated MRI-Compatible Robotic System for MRI-Guided Prostate Intervention," IEEE ASME Trans. Mechatron. 18(1), 273-284 (2012).

A. Krieger, C. Csoma, I.I. Iordachital, P. Guion, A.K. Singh, G. Fichtinger, and L.L. Whitcomb, "Design and preliminary accuracy studies of an MRI-guided transrectal prostate intervention system," Med Image Comput Comput Assist Interv 10(Pt 2), 59-67 (2007).

E.G. Christoforou, I. Seimenis, E. Andreou, E. Eracleous, and N. V. Tsekos, "A novel, general-purpose, MR-compatible, manually actuated robotic manipulation system for minimally invasive interventions under direct MRI guidance," Int. J. Med. Robot. Comput. Assist. Surg. 10(1), 22-34 (2014).

D. Stoianovici, C. Kim, G. Srimathveeravalli, P. Sebrecht, D. Petrisor, J. Coleman, S.B. Solomon, and H. Hricak, "MRI-safe robot for endorectal prostate biopsy," IEEE/ASME Trans. Mechatronics 19(4), 1289-1299 (2014).

Y. Chen, K.-W. Kwok, and Z.T.H. Tse, "An MR-conditional high-torque pneumatic stepper motor for MRI-guided and robot-assisted intervention," Ann. Biomed. Eng. 42(9), 1823-33 (2014).

G.S. Fischer, I. Iordachita, C. Csoma, J. Tokuda, S.P. DiMaio, C.M. Tempany, N. Hata, and G. Fichtinger, "MRI-Compatible Pneumatic Robot for Transperineal Prostate Needle Placement," IEEEASME Trans. Mechatronics 13(3), 295-305 (2008).

20 R. Seifabadi, F. Aalamifar, I. Iordachita, and G. Fichtinger, "Toward teleoperated needle steering under continuous MRI guidance for prostate percutaneous interventions," Int. J. Med. Robot. (Epub ahead of print), 1-15 (2015). 
B. Yang, S. Roys, U.-X. Tan, M. Philip, H. Richard, R. Gullapalli, and J.P. Desai, "Design, Development, and Evaluation of a Master-Slave Surgical System for Breast Biopsy under Continuous MRI," Int. J. Rob. Res. 33(4), 616-630 (2014).

B. Iranpanah, M. Chen, A. Patriciu, and S. Sirouspour, "A Pneumatically Actuated Target Stabilization Device for MRI-Guided Breast Biopsy," IEEE/ASME Trans. Mechatronics 20(3), 1288-1300 (2015).

A. Melzer, B. Gutmann, T. Remmele, R. Wolf, A. Lukoscheck, M. Bock, H. Bardenheuer, and $\mathrm{H}$. Fischer, "INNOMOTION for Percutaneous Image-Guided Interventions," Eng. Med. Biol. Mag. IEEE 27(3), 66-73 (2008).

F. Riillo, C. Bagnato, A.G. Allievi, A. Takagi, L. Fabrizi, G. Saggio, T. Arichi, and E. Burdet, "A Simple fMRI Compatible Robotic Stimulator to Study the Neural Mechanisms of Touch and Pain," Ann. Biomed. Eng. (Epub ahead of print), 1-11 (2016).

E. Franco, D. Brujic, M. Rea, W.M. Gedroyc, and M. Ristic, "Needle-Guiding Robot for Laser Ablation of Liver Tumors Under MRI Guidance," IEEE/ASME Trans. Mechatronics 21(2), 931-944 (2016).

N. Hata, S.-E. Song, O. Olubiyi, Y. Arimitsu, K. Fujimoto, T. Kato, K. Tuncali, S. Tani, and J. Tokuda, "Body-mounted robotic instrument guide for image-guided cryotherapy of renal cancer," Med. Phys. 43(2), 843-853 (2016).

M. Moche, D. Zajonz, T. Kahn, and H. Busse, "MRI-guided procedures in various regions of the body using a robotic assistance system in a closed-bore scanner: preliminary clinical experience and limitations," J Magn Reson Imaging 31(4), 964-974 (2010).

M. D'Onofrio, R. De Robertis, E. Demozzi, S. Crosara, S. Canestrini, and R. Pozzi Mucelli, "Liver volumetry: Is imaging reliable? Personal experience and review of the literature," World J. Radiol. 6(4), 62-71 (2014).

M.M. Arnolli, N.C. Hanumara, M. Franken, D.M. Brouwer, and I.A.M.J. Broeders, "An overview of systems for CT- and MRI-guided percutaneous needle placement in the thorax and abdomen," Int. J. Med. Robot. 11(4), 458-475 (2015).

Determination of Signal-to-Noise Ratio (SNR) in Diagnostic Magnetic Resonance Imaging (NEMA Standard Publication MS 1-2008, 2008). 